\title{
Modifications of Computational Formulae of Decibel Algebra Applied in Acoustics
}

\author{
W. BATKO* \\ Department of Mechanics and Vibroacoustic, University of Science and Technology \\ al. A. Mickiewicza 30, 30-059 Kraków, Poland
}

\begin{abstract}
The analysis of description rules of acoustic effects in decibel algebra was performed in the presented paper. The attention was drawn to the incompatibility between mappings examined on the decibel values set and axioms of the real numbers theory. The consequences of this fact as well as the needs of the necessary modifications of relations describing a cooperation of sound levels from various sources (expressed by adding, subtracting or multiplying) were discussed. The necessary corrections were proposed. The analysis of differences was performed in relation to generally applied rules of processing the decibel measurement results in acoustic examinations.
\end{abstract}

PACS: 43.50.Rq, 43.50.Sr, 43.50.Gf

\section{Introduction}

The majority of acoustic phenomena occurring in the determined spatial-time conditions as well as processes investigated in environmental acoustics [1], are being analysed within a decibel numbers set. Their values are described by a variability of acoustic pressure levels $L_{i}$ $[\mathrm{dB}] ; i=1,2, \ldots, n$ characterizing the analysed effect. They quantify, in accordance with the Weber-Fechner law, related to them acoustic stimuli sensed by humans. They are proportional to the logarithms of the acoustic wave pressure changes quotient versus the assumed for them references.

Their values

$$
L=10 \log \left(\frac{p^{2}}{p_{0}^{2}}\right)[\mathrm{dB}]
$$

define values of 10 common logarithms, from the ratio of the acoustic pressure square $p(t)$ (causing the analysed stimulus) to the reference pressure: $p_{0}=2 \times 10^{-5} \mathrm{~Pa}$, being the threshold of hearing of $1000 \mathrm{~Hz}$ frequency sounds. The process of the mathematical model identification of the acoustic effect being of interest for the researcher is the most often realised on sets of such values: $L_{i}[\mathrm{~dB}]$; $i=1,2, \ldots, n$.

However, using such data sets in the identification process often introduces indiscernible problems [2]. They can be related to decibel algebra deficiencies, it means to the operations of additions and subtractions of sound levels (defined in decibel numbers) determining conditions of their cooperation [3-5].

The lack of a broader methodological reflection related to this problem constituted the inspiration for this paper. The deficiencies of the applied computational formulae

\footnotetext{
* e-mail: batko@agh.edu.pl
}

are given. They concern universally applied formulae of adding and subtracting sound levels as well as - resulting from them - sound levels averaging, or duplicating levels of $n$-sound sources as not fulfilling the real number theory axiomatics. Computational problems corresponding to these facts constitute the grounds for presenting propositions necessary for their corrections.

\section{Theoretical bases of decibel algebra applied in environmental acoustics}

The main calculating relations of algebra, based on decibel values, in the analysis of acoustic effects occurring in environment and described by sound level values $L_{i}$ $[\mathrm{dB}] ; i=1,2, \ldots, n$ is the operation of adding sounds of two noise sources $A$ and $B$ of levels

$$
L_{1}=10 \log \left(\frac{p_{1}^{2}}{p_{0}^{2}}\right)
$$

and

$$
L_{2}=10 \log \left(\frac{p_{2}^{2}}{p_{0}^{2}}\right)
$$

corresponding to generating them pressure disturbances: $p_{1}(t)$ and $p_{2}(t)$.

It is given by the dependence

$$
A+B: L_{1}+L_{2}=10 \log \left(10^{0.1 L_{1}}+10^{0.1 L_{2}}\right) .
$$

The addition operation, defined in such a way, results from the superposition of averaged squares of pressure changes $p_{1}(t)$ and $p_{2}(t)$ generated by those sources

$$
\begin{gathered}
\left\langle p^{2}\right\rangle=\left\langle\left(p_{1}+p_{2}\right)^{2}\right\rangle=\left\langle p_{1}^{2}\right\rangle+2\left\langle p_{1} p_{2}\right\rangle+\left\langle p_{2}^{2}\right\rangle \\
=\left(\left\langle p_{1}^{2}\right\rangle+\left\langle p_{2}^{2}\right\rangle\right)\left(1+\frac{2\left\langle p_{1} p_{2}\right\rangle}{\left\langle p_{1}^{2}\right\rangle+\left\langle p_{2}^{2}\right\rangle}\right) .
\end{gathered}
$$

Waves of noise sources energy, transformed to sound levels values by their reference to the pressure square value $\left(p_{0}^{2}\right)$ and by finding logarithms and multiplying 
by 10 , leads to the equation showing superposition of their influences

$$
\begin{aligned}
& 10 \log \left(\frac{p^{2}}{p_{0}^{2}}\right)=10 \log \left(\frac{p_{1}^{2}}{p_{0}^{2}}+\frac{p_{2}^{2}}{p_{0}^{2}}+\frac{2 p_{1} p_{2}}{p_{0}^{2}}\right) \\
& =10 \log \left(\left(\frac{p_{1}^{2}}{p_{0}^{2}}+\frac{p_{2}^{2}}{p_{0}^{2}}\right)\left(1+\frac{2 p_{1} p_{2}}{p_{1}^{2}+p_{2}^{2}}\right)\right) \\
& =10 \log \left(\frac{p_{1}^{2}}{p_{0}^{2}}+\frac{p_{2}^{2}}{p_{0}^{2}}\right)+10 \log \left(1+\frac{2 p_{1} p_{2}}{p_{1}^{2}+p_{2}^{2}}\right) .
\end{aligned}
$$

This equation is simplifying at the assumption of incoherence of wave influences of noise sources $A$ and $B$, at which it is possible to omit the coherence component $10 \log \left(1+\frac{2 p_{1} p_{2}}{p_{1}^{2}+p_{2}^{2}}\right)$, which is adopting the zero value. Components $\frac{p_{1}^{2}}{p_{0}^{2}}$ and $\frac{p_{2}^{2}}{p_{0}^{2}}$ occurring in this relation can be expressed by related to them pressure levels $L_{1}$ and $L_{2}$ :

$$
\frac{p_{1}^{2}}{p_{0}^{2}}=10^{0.1 L_{1}}
$$

and

$$
\frac{p_{2}^{2}}{p_{0}^{2}}=10^{0.1 L_{2}}
$$

at which the defined dependence (4) for adding sound levels of two noise sources $A$ and $B$ of levels $L_{1}$ and $L_{2}$ is obtained.

Utilization of the definition (4) of addition sound levels expressed by decibel numbers - assumed in acoustic investigations - constitutes the bases (generally present in analyses and investigations of environmental noises) of formulae concerning: sound levels subtraction (9), their averaging (10) and the assessment of effects of cooperation of $n$ equivalent sound sources (11):

$$
\begin{aligned}
& A-B: L_{1}-L_{2}=10 \log \left(10^{0.1 L_{1}}-10^{0.1 L_{2}}\right), \\
& \bar{A}: \bar{L}=10 \log \left(\frac{1}{n} \sum_{i=1}^{n} 10^{0.1 A_{i}}\right), \\
& k \times A: k \times L=L+10 \log k .
\end{aligned}
$$

Defined in such a way algebraic operations for processing the acoustic investigations results - expressed by decibel numbers - are not in conformity with the axiomatic theory of real numbers since it requires from the adding operation the fulfilment of the following rules:

- $x_{1}, x_{2} \in X ; x_{1}+x_{2} \in X$.

- There is such element 0 that for each $x \in X$ the relation $x+0=x$ is satisfied (Archimedes principle).

- There is element $(-x)$ called opposite to $x$ such that: $x+(-x)=0$.

- $x_{1}+x_{2}=x_{2}+x_{1}$ (commutative rule).

Especially it does not meet the requirements of the Archimedes principle; however it is usually passed with- out a wider reflection concerning its effects in acoustic calculations.

\section{Correction of computational relations of decibel algebra applied in processing of acoustic investigations results}

The basic defect of the computational formula concerning the addition operation of acoustic pressures determined by the decibel measure (with which one is dealing with in acoustic investigations) is its incompatibility with the Archimedes principle. This leads to the necessity of excluding such investigation situation, in which there are sources of acoustic pressure equal to zero. It renders difficult - in certain situations - constructing the model approximating the results of acoustic measurements. There is no logical justification of the situation, in which addition of two sources of zero level acoustic pressures generates a result of $3 \mathrm{~dB}$.

An omission of sources of acoustic pressures being zero can correspond to difficulties in the automation process of experiments and identification calculations in environmental acoustics. A significant premise of the need of correction of the currently applied computational procedures is also the requirement of assessing the uncertainty of the performed acoustic experiments $[6,7]$. It will impose the necessity of assessing possible numerical errors related to processing data bases of results of sound levels estimating them in situations when the arithmetic applied to the measurement results set is not fully correctly defined.

The above mentioned reasons prompted investigations in this field. They allowed to formulate indispensable corrections of the decibel algebra calculations used in acoustic investigations of the environment. They were derived by the modification of the equation for adding two levels of acoustic pressures.

In the proposed form it is determined by the following equation:

$$
A+B: L_{1}+L_{2}=10 \log \left(10^{0.1 L_{1}}+10^{0.1 L_{2}}-1\right)
$$

The correction of relation (4) describing superposition of acoustic pressure levels of two sources $A$ and $B$ is done by decreasing the number, whose logarithm is being found (it means the sum of relative energy of acoustic waves

$$
E_{A}=\frac{p_{A}^{2}}{p_{0}^{2}}=10^{0.1 L_{1}}
$$

and

$$
E_{B}=\frac{p_{B}^{2}}{p_{0}^{2}}=10^{0.1 L_{2}}
$$

by the value of a single unit

$$
E_{0}=\frac{p_{0}^{2}}{p_{0}^{2}},
$$

which corresponds to the acoustic pressure level equal to zero dB $\left(10 \log \frac{p_{0}^{2}}{p_{0}^{2}}\right)$.

The corrected form - in the case of subtracting acoustic pressure levels of sources $A$ and $B-$ is determined 
by the dependence

$$
A-B: L_{1}-L_{2}=10 \log \left(10^{0.1 L_{1}}-10^{0.1 L_{2}}+1\right)
$$

The generalised addition operation related to the negative pressure level of one of the sources can be the relation

$$
\begin{aligned}
A & +B: L_{1}+L_{2} \\
& =10 \log \left(10^{0.1 L_{1}}+\operatorname{sgn}\left(L_{2}\right) \times 10^{0.1 L_{2}}-1\right),
\end{aligned}
$$

in which $\operatorname{sgn}\left(L_{2}\right)$ is determined by

$$
\operatorname{sgn}\left(L_{2}\right)=\left\{\begin{array}{l}
1 \text { when } L_{2} \geq 0, \\
-1 \text { when } L_{2}<0 .
\end{array}\right.
$$

The assumed above correction of addition operation generates changes in formulae related to: averaging acoustic pressures (10), or the assessment of effects of cooperation of $n$ equivalent sound sources (11).

Within the assumed modification they are determined by (19) and (20):

$$
\begin{aligned}
& L_{a v}=10 \log \left(\frac{1}{n} \sum_{i-1}^{n} 10^{0.1 L_{i}}-\left(1-\frac{1}{n}\right)\right), \\
& k \times L=L+10 \log \left(1-\frac{1}{k}\right) .
\end{aligned}
$$

A certain numerical error generated by operations of adding, subtracting, averaging of acoustic level pressures (10) or assessments of effects of cooperation of $n$ equivalent sound sources (11) can be attributed to the currently applied algebra of processing the pressure level results. It is determined by the difference of the results obtained when the current computational formulae are used $\delta^{c}$, i.e. Eqs. (4), (9), (10) and (11):

$$
\delta_{j}=\delta_{j}^{\mathrm{m}}-\delta_{j}^{\mathrm{c}}, \quad j=1,2, \ldots, 4
$$

and the results obtained by the proposed modifications $\delta^{\mathrm{m}}$, i.e. Eqs. (13), (17), (20) and (21).

They can be estimated according to formulae given in Table.

\section{TABLE}

Relations determining errors of the current formulae of the decibel algebra.

\begin{tabular}{c|l|l}
\hline \hline No. & \multicolumn{1}{|c|}{ Operation } & Correction $\delta_{j} ; j=12, \ldots, 4$ \\
\hline 1 & addition: $A+B$ & $10 \log \left(1-1 /\left(10^{0.1 A}+10^{0.1 B}\right)\right)$ \\
2 & subtraction $A-B$ when $(A \neq B)$ & $10 \log \left(1+1 /\left(10^{0.1 A}+10^{0.1 B}\right)\right)$ \\
3 & duplication $k \times A$ & $10 \log \left(1-\left(1-\frac{1}{K}\right) 10^{-0.1 A}\right)$ \\
4 & summation of $n$ sources & $10 \log \left(1-(n-1) / \sum_{i=1}^{n} A_{i}\right)$ \\
5 & averaging & $10 \log \left(1-\left(1-\frac{1}{n}\right) / \sum_{i=1}^{n} 10^{0.1 A_{i}}\right)$
\end{tabular}

\section{Conclusions}

Deficiencies of the decibel algebra applied in processing the acoustic investigation results are discussed in the paper. They indicated the need for the modification of the existing and currently applied computational formulae for: sum and difference of acoustic pressure levels, averaging of the set of such results as well as superposition of $n$ equivalent sound sources.

The necessary corrections were proposed. The assessment of the possible numerical errors, generated by the current processing calculus of the decibel numbers describing the acoustic phenomenon being under investigation, was performed.

The present progress of numerical techniques, as well as the development of automation of metrological processes and the way of obtaining acoustic data bring about the need of taking into consideration uncertainties of numerical calculations in the research [2]. The results of this paper are essential for this task, since they can be used for attempting to develop the axiomatics for identification experiments in the acoustics domain determined by the acoustic pressure level measurements, at the selection of the distance metric, useful for comparisons.

This field of research, being in the author's sphere of interests, will be undertaken in the nearest future.

\section{References}

[1] Directive 2002/49/WE of the European Parliament and of the Council of 25 June 2002, related to the assessment and management of environmental noise, Official Journal of the European Communities 18.07.2002.

[2] W. Batko, Acta Phys. Pol. A 118, 7 (2010).

[3] H.J. Bress, Introduction and basic concepts of noise quality evaluation, 2nd ed., K. Larsen \$ Son A/S, DK 2600 Glostrup, 1987 (in German). 
[4] H. Hoffman, A. Luepke, 0 Dezibel+0 Dezibel=3 Dezibel, Erich Schmidt Verlag, Berlin 1975.

[5] J.H. Maue, 0 Decibel+0 Decibel=3 Decibel. Decibel numbers calculus, mathematical relations in sets of decibel numbers, Erich Schmidt Verlag, Berlin 2009.

[6] W. Batko, R. Bal-Pyrcz, Arch. Acoust. 31, (supplement), 253 (2006).
[7] International Organization for Standardization, Guide to the Expression of Uncertainty in Measurements, 1995. 